\title{
Impact of Patient Counseling and Socioeconomic Factors on Initiation of Rehabilitation Program in Spinal Cord Injury Patients Presenting to a Tertiary Spine Unit in India
}

\author{
Siddharth Narasimhan Aiyer ${ }^{1}$, Vignesh Gunasekaran ${ }^{2}$, Latha Mani ${ }^{2}$, Sri Vijay Anand K. S. , \\ Shanmuganathan Rajasekaran ${ }^{1}$, Ajoy Prasad Shetty ${ }^{1}$ \\ ${ }^{1}$ Department of Spine Surgery, Ganga Hospital, Coimbatore, India \\ ${ }^{2}$ Ganga Spine Injury Rehabilitation Centre, Coimbatore, India
}

\section{Study Design: Prospective case series.}

Purpose: This study aimed to investigate the impact of education, financial income, occupation, and patient counseling on the timing of enrolment in a spinal cord injury (SCI) rehabilitation program.

Overview of Literature: A rehabilitation program following SCI is essential to improve functional outcomes. Socioeconomic factors can affect the timing of enrolment to a rehabilitation program. Literature on the effects of socioeconomic factors among patients with $\mathrm{SCl}$ in the Indian scenario is limited.

Methods: A prospective, consecutive analysis of patients with SCI was performed with 1-year follow-up. Assessment of the timing of enrolment to a rehabilitation program was performed using the modified Kuppuswamy socioeconomic scores (MKSS). Patients admitted to the SCl unit (group A), underwent intensive individual, group, and family counseling sessions to encourage early enrolment into a rehabilitation program. Patients presenting directly for rehabilitation (group B) were analyzed for comparison.

Results: A total of 153 patients were recruited. Group A was composed of 122 patients who started the rehabilitation program after a mean of 28 days, compared with a mean of 149 days for 31 patients in group B. In group A, 104 patients (85\%; mean MKSS, 14.02) and 18 patients (15\%; mean MKSS, 15.61) enrolled for rehabilitation $<6$ weeks and $\geq 6$ weeks, respectively. In group $B, 12$ patients (39\%; mean MKSS, 13.69) and 19 patients (61\%; mean MKSS, 12.10) enrolled for rehabilitation $<6$ weeks and $\geq 6$ weeks, respectively. The total MKSS and scores for education, income, and occupation did not show a significant difference between the two both groups $(p>0.05)$.

Conclusions: Early patient counseling in the acute care unit helps in the early enrolment of patients with poor socioeconomic demographic profile to a rehabilitation program.

Keywords: Rehabilitation; Spinal cord injuries; Socioeconomic factors; Counseling 


\section{Introduction}

The estimated incidence rates of spinal cord injury (SCI) ranged from 10 to 80 per million per year, with global prevalence rates of $223-1,000$ per million population [17]. These estimates of the worldwide incidence have significant limitations because of the inadequacy of national registry databases for SCI in many countries in Asia and Africa $[1,3,8,9]$. In the absence of high-quality epidemiology studies pertaining to SCI in India, an accurate estimate of the incidence and prevalence of SCI is difficult to obtain [9-11]. However, hospital-based pilot data suggest that the burden of SCI is substantial, and the demographics in India widely varied from global reports [8,12-14].

In developing countries like India, where the per capita income is low and a sizeable proportion of the population is located below the poverty line, access to rehabilitation facilities may be limited by financial constraints [15]. Data are limited about the impact of socioeconomic factors on enrolment in rehabilitation programs for patients with SCI in developing countries like India. The social stigma associated with SCI and lower educational levels of patients may have additional impacts on the initiation of a timely rehabilitation program. Patients with SCI have substantially higher rates of unemployment, which preclude universal access to rehabilitation programs $[15,16]$.

The initiation of an early rehabilitation program for traumatic and non-traumatic SCI has shown improved functional outcomes and independence measures for given levels of neurological injury $[11,17,18]$. Patients with SCI who participate in early rehabilitation and work in reintegration programs also demonstrate a reduced risk of bankruptcy [19].

Considering the increasing incidence of SCI in India, condition's enormous associated costs, inadequate epidemiology data pertaining to India, and beneficial effects of early rehabilitation, this prospective study aimed to analyze the effects of the socioeconomic factors of occupation, education, income, and patient counseling on the timing of patient enrolment in a dedicated SCI rehabilitation unit following discharge from an acute care unit.

\section{Materials and Methods}

This study was conducted at Ganga Hospital, Coimbatore, India which is a tertiary spine trauma unit with a dedicated post-SCI rehabilitation center. A prospective analysis was performed for consecutive patients with SCI who presented to the rehabilitation unit over a 12-month period from August 2015 to July 2016. This study was approved by the Institutional Review Board (approval no., IRB 2015/07/03), and all applicable institutional and governmental regulations concerning the ethical use of human volunteers were followed during the course of this research. Informed consent was obtained from all patients participating in the study.

Demographic details, mode of injury, neurological presentation at injury, time interval between the injury and rehabilitation initiation, and type of SCI (traumatic versus non-traumatic SCI) were assessed. Demographic variables included age, sex, and marital status at the time of SCI. The mode of injury in traumatic SCI and the etiology of non-traumatic SCI were also documented. A neurological assessment of SCI was performed based on the American Spinal Injury Association (ASIA) scale at the time of injury.

The patients were segregated into two groups: Group A consisted of patients undergoing primary acute trauma care for a spinal injury at our institute. Group A patients were counseled to consider early rehabilitation once they were fit for discharge from the acute trauma care facility. Discharge to the rehabilitation unit was considered once the condition of the patient met the criteria for discharge: wound healing was satisfactory to pursue rehabilitation training for independent log rolling and wheelchair transfers. Patients who had suffered an SCI in a clinical polytrauma setting were assessed collectively with a multidisciplinary team discussion. Patients who were considered fit to pursue rehabilitation training and assisted mobilization were enrolled in the rehabilitation unit. Patients with cervical SCI were assessed for respiratory function, and patients able to maintain adequate oxygen saturation on room air or with the minimum requirement of tracheostomy care were considered for transfer.

Three counseling sessions of approximately 20 minutes each were conducted by a rehabilitation team that included a spine surgeon, clinical psychologist, physiotherapist, and rehabilitation medicine physician. The patients underwent three sessions to provide insight into the nature of the injury, goals for the rehabilitation program, and methods used to prevent pressure sores. Three counseling sessions were conducted in the form of (1) individual counseling, (2) group counseling in a group of 2-3 patients, and (3) family counseling for the caregivers. 
Group B consisted of patients primarily presenting directly for the SCI rehabilitation program following acute SCI care. Each patient was assessed individually by the authors (S.R. and S.A.) to determine the adequacy of the primary care introduced. This assessment included the adequacy of any decompression/stabilization procedure performed. If the treatment was considered satisfactory, the patient was enrolled in the study. The primary treating center for group B included a district level public hospital, state medical university hospitals, and private hospitals.

Any patient counseling sessions received by group B patients during their primary treatment at other institutes were documented. Pressure sores noted at presentation and any new pressure sores were documented during the follow-up period for 1 year in both groups. The residential

Table 1. Modified Kuppuswamy Scale with the income modified for 2012

\begin{tabular}{|c|c|}
\hline & Score \\
\hline \multicolumn{2}{|l|}{ Education } \\
\hline Profession or honours & 7 \\
\hline Graduate or post graduate & 6 \\
\hline Post high school diploma & 5 \\
\hline High school & 4 \\
\hline Middle school & 3 \\
\hline Primary school & 2 \\
\hline Illiterate & 1 \\
\hline \multicolumn{2}{|l|}{ Income in rupees } \\
\hline$\geq 31,507$ & 12 \\
\hline $15,754-31,506$ & 10 \\
\hline $11,817-15,753$ & 6 \\
\hline $7,878-11,816$ & 4 \\
\hline $4,727-7,877$ & 3 \\
\hline $1,590-4,726$ & 2 \\
\hline$\leq 1,589$ & 1 \\
\hline \multicolumn{2}{|l|}{ Occupation } \\
\hline Professional & 10 \\
\hline Semi-professional & 6 \\
\hline Clerical, shop-owner, farmer & 5 \\
\hline Skilled worker & 4 \\
\hline Semi-skilled worker & 3 \\
\hline Unskilled worker & 2 \\
\hline Unemployed & 1 \\
\hline
\end{tabular}

Total score 26-29, upper class; total score 16-25, upper middle class; total score 11-15, lower middle class; total score 5-10, upper lower class; total score $<5$, lower class. address and transportation distance to reach the acute spine trauma unit and rehabilitation center were also noted for both groups.

The patients were assessed to identify their socioeconomic backgrounds using the widely used and validated modified Kuppuswamy score (MKSS) [15,20], as shown in Table 1. The patients were evaluated based on their scores for monthly family income, education, and occupation, and a combined total score was calculated to reflect their socioeconomic status using the MKSS. The updated income based on the 2012 modification reported by Bairwa et al. [20] was utilized.

The subgroup analysis of the MKSS for both groups was carried out based on the time interval ( $<6$ weeks versus $\geq 6$ weeks) between injury onset and admission to the rehabilitation center. Statistical analyses were performed using IBM SPSS software ver. 23.0 (IBM Corp., Armonk, NY, USA). Results were expressed in mean and standard deviations. The paired $t$-test and chi-square test were used for analysis. A p-value less than 0.05 was considered to be statistically significant.

\section{Results}

A total of 153 consecutive, prospective patients were enrolled in this study, of which 138 had traumatic SCI and 15 had non-traumatic SCI.

\section{Traumatic spinal cord injury}

The mean age of the patients with traumatic SCI was $37.8 \pm 14.9$ years (range, $13-80$ years). There were 128 male and 10 female patients in the traumatic SCI group. At the time of injury, 96 patients (69\%) were married. The leading mode of injury in the traumatic SCI group was a fall from a height in 63 patients $(45.6 \%)$, followed by road traffic accidents in 62 patients (44.9\%). Other modes of injury were diving-related injuries in four patients $(2.8 \%)$, a heavy object falling on the spine in four patients $(2.8 \%)$, unprovoked animal attacks (wild elephants, $n=2$; bull, $\mathrm{n}=1$ ) in three patients $(2.1 \%)$, and SCI following a shrapnel injury from a bomb explosion in one patient $(0.72 \%)$.

The patients were assessed based on their level of injury and segregated into cervical (C1-7), thoracic (T1-9), and thoracolumbar (T10-L2) injuries. The mode of injury, level of injury, and ASIA scale for traumatic SCI are presented in Table 2. Cervical injuries were more com- 
Table 2. Traumatic spinal cord injury patients, details of region of injury, and American Spinal Injury Association score at presentation

\begin{tabular}{|c|c|c|c|c|c|c|c|c|c|c|c|c|}
\hline \multirow{2}{*}{ Variable } & \multicolumn{4}{|c|}{ Cervical (C1-C7, N=48) } & \multicolumn{4}{|c|}{ Thoracolumbar ( $\mathrm{T} 10-\mathrm{L} 2, \mathrm{~N}=66)$} & \multicolumn{4}{|c|}{ Thoracic (T1-T9, N=23) } \\
\hline & $A$ & B & C & $\mathrm{D}$ & A & B & C & $\mathrm{D}$ & A & B & C & D \\
\hline Fall from height $(\mathrm{N}=63)$ & 11 & - & 2 & 1 & 26 & 2 & 11 & - & 10 & - & - & - \\
\hline Road traffic accident ( $\mathrm{N}=62$ ) & 17 & 1 & 8 & 4 & 20 & 1 & - & - & 9 & 1 & 1 & - \\
\hline Diving related injury ( $\mathrm{N}=4$ ) & 3 & - & - & - & - & - & - & - & 1 & - & - & - \\
\hline Animal attacks ( $\mathrm{N}=3$ ) & - & 1 & - & - & 2 & - & - & - & - & - & - & - \\
\hline Fall of heavy object on spine $(\mathrm{N}=4)$ & - & - & - & - & 3 & - & - & - & 1 & - & - & - \\
\hline Bomb explosion ( $\mathrm{N}=1)$ & - & - & - & - & 1 & - & - & - & - & - & - & - \\
\hline
\end{tabular}

Table 3. Etiology for non-traumatic SCl

\begin{tabular}{lc} 
Etiological cause for $\mathrm{SCI}$ & No. of cases \\
\hline Cervical myelopathy & 2 \\
\hline Intra-dural tumor related deficit & 2 \\
\hline Pyogenic infective spondylodiscitis & 4 \\
\hline Tuberculosis of spine & 1 \\
\hline Spinal kyphotic deformity & 3 \\
\hline Disc prolapse & 1 \\
\hline Thoracic myelopathy due to ossified ligamentum flavum & 2 \\
\hline
\end{tabular}

SCl, spinal cord injury.

mon in road traffic accidents, and thoracolumbar injuries were more likely to result from a fall from a great height. In traumatic SCI, thoracolumbar injuries 66 patients (47.8\%) were most common, followed by cervical injuries in 48 patients $(34.7 \%)$ and thoracic injuries in 23 patients (16.6\%).

Falls from a great height resulted in thoracolumbar injury in $61.9 \%$, cervical injury in $22.2 \%$, and thoracic injury in $15.8 \%$ of the patients. In patients with SCIs caused by road traffic accidents, $48.3 \%$ had cervical injuries, $33.8 \%$ had thoracolumbar injuries, and $17.7 \%$ had thoracic spine injuries. A neurological assessment documented that $75.3 \%$ of the patients had ASIA A impairments, $4.3 \%$ had ASIA B deficits, 15.9\% demonstrated ASIA C impairment, and $3.6 \%$ had ASIA D impairment.

\section{Non-traumatic spinal cord injury}

Fifteen patients presented with SCI following a nontraumatic etiology. The average age in the non-traumatic group was $44.2 \pm 18.7$ years (range, $10-70$ years), with four female and 11 male patients. The etiological causes of neurological dysfunction are shown in Table 3.

\section{Analysis for group A}

Group A was composed of 122 patients undergoing acute treatment for SCI at treatment for SCI at Ganga Hospital, Coimbatore, India. The average delay between the injury onset and initiation of a rehabilitation program was $28 \pm 30$ days. Moreover, 104 patients (85.2\%) enrolled within 6 weeks of injury, and 18 patients had delayed enrolment of $\geq 6$ weeks from their injury. The average distance between the place of residence and the institute was $180 \pm 165 \mathrm{~km}$ (range, 10-1,200 km). In group A, 10 of 122 patients developed pressure sores during the follow-up period.

\section{Analysis for group B}

Group B was composed of 31 patients who presented for enrolment to a rehabilitation program following primary treatment for SCI at other medical institutes. The patients had an average delay of $149 \pm 168$ days between their injury onset and the initiation of a rehabilitation program. Moreover, 12 patients enrolled for rehabilitation within 6 weeks of injury, and 19 patients enrolled $\geq 6$ weeks following injury. The average distance of the rehabilitation center from the patients' place of residence was $321 \pm 345$ $\mathrm{km}$ (range, 15-2,100 km). In this group, 17 patients had pressure sores at the time of presentation or in the past prior to rehabilitation enrolment.

\section{Timing of enrolment in group $A$ and $B$}

The chi-square test for assessment of timing for enrolment 
Table 4. Modified Kuppuswamy scores for group A and B

\begin{tabular}{lccc} 
Variable & $<6$ wk & $\geq 6$ wk & $p$-value \\
Group A & & & \\
\hline No. of patients & 104 & 18 & \\
\hline Education & $4.08 \pm 1.42$ & $3.94 \pm 1.87$ & 0.71 \\
\hline Income & $5.46 \pm 3.47$ & $5 \pm 2.72$ & 0.40 \\
\hline Occupation & $4.47 \pm 2.44$ & $6.66 \pm 4.03$ & 0.18 \\
\hline Total & $14.02 \pm 5.77$ & $15.6 \pm 7.34$ & 0.30 \\
\hline Group B & & & \\
\hline No. of patients & 12 & 19 & \\
\hline Education & $4.41 \pm 1.31$ & $4.26 \pm 2.32$ & 0.84 \\
\hline Income & $6.41 \pm 4.39$ & $4.05 \pm 3.47$ & 0.10 \\
\hline Occupation & $3.08 \pm 1.88$ & $3.78 \pm 3.24$ & 0.50 \\
\hline Total & $13.9 \pm 5.35$ & $12.1 \pm 7.51$ & 0.40 \\
\hline
\end{tabular}

Values are presented as number or mean \pm standard deviation.

Table 5. Modified Kuppuswamy scores for groups A and B

\begin{tabular}{lc} 
Variable & Score \\
Group A & \\
\hline Upper class & 7 \\
\hline Upper middle class & 43 \\
\hline Lower middle class & 35 \\
\hline Upper lower class & 35 \\
\hline Lower class & 2 \\
\hline Group B & \\
\hline Upper class & 2 \\
\hline Upper middle class & 9 \\
\hline Lower middle class & 6 \\
\hline Upper lower class & 13 \\
\hline Lower class & 1 \\
\hline
\end{tabular}

( $<6$ weeks versus $\geq 6$ weeks) between group A and group B showed a significant difference, with group A showing a higher patient enrolment within 6 weeks from injury $(p<0.05)$.

\section{Socioeconomic evaluation}

The socioeconomic profile of groups A and B with the MKSS is highlighted in Tables 4-6. In both groups, the total MKSS and individual scores for education, income, and occupation were comparable between early $(<6$ weeks) and delayed ( $\geq 6$ weeks) rehabilitation with no sig- nificant difference. None of the patients in group B had attended previous formal counseling sessions to discuss the nature of their injury, to describe the need for rehabilitation, or to outline goals for social reintegration.

\section{Discussion}

The economic costs of treatment for SCI are enormous and have been estimated to be between $\$ 100,000$ and $\$ 500,000$ in the first year [21-25]. Considering the high costs involved with SCI, socioeconomic status has an important bearing on the treatment sought by the patients [15]. However, given the absence of a national registry, the impact of these factors on the initiation of a rehabilitation program has not been studied previously in an Indian context [15].

The patient and the family underwent counseling to encourage early initiation of a rehabilitation program following discharge from the acute trauma unit. In group A, 104 patients $(85.2 \%)$ started the rehabilitation program within 6 weeks compared to 12 patients (38.7\%) in group B. The lack of dedicated units to undertake SCI rehabilitation is evident on the average distance of residence from the rehabilitation unit for group A and group B, i.e., 180 $\mathrm{km}$ and $321 \mathrm{~km}$, respectively. The findings of the study suggest that immediate transfer to the rehabilitation unit following acute surgical care can aid in the initiation of a rehabilitation program. Discharge of the patient following acute care to a non-medical facility/home makes subsequent initiation of a rehabilitation program difficult. This may be a possible reason because the average delay before rehabilitation was started in group B was 149 days compared with 28 days for group A.

In this study, socioeconomic factors were assessed using the MKSS. As the financial costs of SCI treatment are high, poor socioeconomic factors can delay the rehabilitation process, resulting in poor reintegration into the society [15]. Chhabra and Bhalla [15] reported on access to various SCI management facilities based on financial constraints, which were assessed by the MKSS. The authors noted that all classes, except the upper class, had limited access to SCI management avenues in India. We noted that the scores for education, occupation, and income in both groups were comparable in this study (Table 6). The authors noted that $60 \%$ of the patients in group A and $64 \%$ patients in group B were in the lower middle or lower class. However, the recruitment for rehabilitation program 
Table 6. Comparison between group A and B

\begin{tabular}{lrcc} 
Variable & Group A (N=122) & Group B (N=31) & $p$-value \\
\hline Education & $4.05 \pm 1.48$ & $4.32 \pm 1.97$ & 0.39 \\
Income & $5.65 \pm 3.57$ & $4.96 \pm 3.96$ & 0.34 \\
\hline Occupation & $4.05 \pm 2.49$ & $3.51 \pm 2.77$ & 0.29 \\
\hline Total score & $14.27 \pm 6.04$ & $12.80 \pm 6.72$ & 0.23 \\
\hline
\end{tabular}

Values are presented as mean \pm standard deviation.

of patients in group A who received specific counseling was statistically higher regardless of the MKSS. In light of these findings, the authors suggest that counseling to encourage early rehabilitation can improve enrolment even in lower-class patients.

Logistical concerns of transportation for a SCI victim over long distances in developing countries can be very challenging. The authors noted that the average distance of the rehabilitation center from the residence in group A was $180 \pm 165 \mathrm{~km}$ compared with $321 \pm 345 \mathrm{~km}$ in group B. In this study, the authors speculate that the logistical concern over transportation may have an impact on the enrolment following SCI. In a study on home visit assessment for 546 patients with SCI, Prabhaka and Thakker [10] noted that patients faced considerable difficulty returning for follow-up after discharge in the first year because of financial and social issues. SCI complications are most frequent in the first year following surgery; thus, the authors advocated "paraplegia safari" where a rehabilitation team comprised of seven members would make home visits in the first year to help reduce the complications and readmission rates following SCI. In this study, group A recorded 10 patients $(8 \%)$ with pressure sores compared with 17 patients (54\%) in group B, suggesting that an early rehabilitation program can reduce this potential complication.

Epidemiological data generated from this prospective series of SCI patients recruited at a tertiary SCI treatment facility is valuable, particularly considering the limited available knowledge regarding SCI in India. A unique contribution toward SCI in southeast Asia arises from a large number of SCI following two-wheeler accidents and falls from heights including trees, rooftops, and construction sites $[1,3]$. These injuries account for the common mode of traumatic SCI in this region, which is reflected in the results of this study. A male preponderance was noted among traumatic SCI patients, with previous stud- ies quoting 4-5 times higher incidence among men [4,21]. This study noted that $92 \%$ of the SCI patients were men. Global epidemiology studies have noted complete injury rates between $40 \%$ and $50 \%$ for America and a trend toward more incomplete injuries in Europe and Australia $[4,21]$. However, this study documented a $75 \%$ prevalence of complete injuries, which is substantially higher than the rates from other geographical regions.

The beneficial effects of a rehabilitation program following SCI are well studied. Sumida et al. [17] reported on a multicenter trial including 123 patients and assessed the timing to begin rehabilitation in three groups: (1) acute, $<2$ weeks; (2) recovery group, 2 weeks to 6 months; and (3) chronic, more than 6 months. The authors concluded that the physical functional independence measure for a given ASIA scale injury improved with early rehabilitation [17]. New [18] reported on 70 patients with non-traumatic SCI and concluded that their disability was significantly reduced with a good rehabilitation program. However, poor awareness among the Indian population with regard to dedicated SCI rehabilitation units is likely to be a substantial constraint in the effective treatment of SCI [9].

This study has a few limitations. Epidemiological data may have had a selection bias, as the institution is a tertiary care spine trauma unit. The predominance of complete injury may result from referral of more complicated SCI to the institution. The socioeconomic profile of patients visiting a public or university hospital may vary considerably from private hospitals, and such study may be limited as the sample population was from a predominantly narrow spectrum of socioeconomic status. India has limited numbers of SCI rehabilitation centers, and the logistical concerns of transportation of SCI patients over long distance may result in a bias in the sample population.

The authors suggest that the effects of poor socioeconomic factors on the initiation of an early rehabilitation program can be partially offset by encouraging early and direct transfer to a rehabilitation facility following discharge from the acute care facility. Moreover, the authors suggest that a combined multidisciplinary team effort consisting of a primary care attending physician, a spine surgeon, a physiotherapist, and a clinical psychologist is vital to ensure early recruitment in a rehabilitation program. The need for transfer to a dedicated, specialized care rehabilitation facility after discharge is often inadequately addressed and needs to be further encouraged. 


\section{Conclusions}

In developing countries like India, where poor socioeconomic factors can impede patient enrolment in an SCI rehabilitation program, early counseling with a multidisciplinary team during acute trauma care admission helps ensure early initiation of a rehabilitation program.

\section{Conflict of Interest}

No potential conflict of interest relevant to this article was reported.

\section{Funding}

The study was funded by Ganga Orthopaedic Research and Education Foundation.

\section{Author Contributions}

Siddharth N. Aiyer: conception and design, data acquisition, analysis of data, drafting of the manuscript; Vignesh Gunasekaran: conception and design, data acquisition, analysis of data; Latha Mani: data acquisition, analysis of data; K. S. Sri Vijay Anand: obtaining funding, administrative support; Shanmuganathan Rajasekaran: critical revision, supervision; and Ajoy Prasad Shetty: conception and design, critical revision, supervision.

\section{References}

1. Cripps RA, Lee BB, Wing P, Weerts E, Mackay J, Brown D. A global map for traumatic spinal cord injury epidemiology: towards a living data repository for injury prevention. Spinal Cord 2011;49:493-501.

2. Fitzharris M, Cripps RA, Lee BB. Estimating the global incidence of traumatic spinal cord injury. Spinal Cord 2014;52:117-22.

3. Lee BB, Cripps RA, Fitzharris M, Wing PC. The global map for traumatic spinal cord injury epidemiology: update 2011, global incidence rate. Spinal Cord 2014;52:110-6.

4. Devivo MJ. Epidemiology of traumatic spinal cord injury: trends and future implications. Spinal Cord 2012;50:365-72.

5. Wyndaele M, Wyndaele JJ. Incidence, prevalence and epidemiology of spinal cord injury: what learns a worldwide literature survey? Spinal Cord 2006;44:523-9.

6. Singh A, Tetreault L, Kalsi-Ryan S, Nouri A, Fehlings MG. Global prevalence and incidence of traumatic spinal cord injury. Clin Epidemiol 2014;6:309-31.

7. Selvarajah S, Hammond ER, Haider AH, et al. The burden of acute traumatic spinal cord injury among adults in the united states: an update. J Neurotrauma 2014;31:228-38.

8. Agarwal P, Upadhyay P, Raja K. A demographic profile of traumatic and non-traumatic spinal injury cases: a hospital-based study from India. Spinal Cord 2007;45:597-602.

9. Chhabra HS, Arora M. Demographic profile of traumatic spinal cord injuries admitted at Indian Spinal Injuries Centre with special emphasis on mode of injury: a retrospective study. Spinal Cord 2012;50:74554.

10. Prabhaka MM, Thakker TH. A follow-up program in India for patients with spinal cord injury: paraplegia safari. J Spinal Cord Med 2004;27:260-2.

11. Pandey V, Nigam V, Goyal TD, Chhabra H. Care of post-traumatic spinal cord injury patients in India: an analysis. Indian J Orthop 2007;41:295-9.

12. Lalwani S, Singh V, Trikha V, et al. Mortality profile of patients with traumatic spinal injuries at a level I trauma care centre in India. Indian J Med Res 2014;140:40-5.

13. Chacko V, Joseph B, Mohanty SP, Jacob T. Management of spinal cord injury in a general hospital in rural India. Paraplegia 1986;24:330-5.

14. Mathur N, Jain S, Kumar N, Srivastava A, Purohit $\mathrm{N}$, Patni A. Spinal cord injury: scenario in an Indian state. Spinal Cord 2015;53:349-52.

15. Chhabra HS, Bhalla AM. Influence of socio-economic status on access to different components of SCI management across Indian population. Spinal Cord 2015;53:816-20.

16. Gupta N, Solomon J, Raja K. Employment after paraplegia in India: a postal survey. Spinal Cord 2011;49:806-11.

17. Sumida M, Fujimoto M, Tokuhiro A, Tominaga T, Magara A, Uchida R. Early rehabilitation effect for traumatic spinal cord injury. Arch Phys Med Rehabil 2001;82:391-5.

18. New PW. Functional outcomes and disability after nontraumatic spinal cord injury rehabilitation: re- 
sults from a retrospective study. Arch Phys Med Rehabil 2005;86:250-61.

19. Hollingworth W, Relyea-Chew A, Comstock BA, Overstreet JK, Jarvik JG. The risk of bankruptcy before and after brain or spinal cord injury: a glimpse of the iceberg's tip. Med Care 2007;45:702-11.

20. Bairwa M, Rajput M, Sachdeva S. Modified Kuppuswamy's socioeconomic scale: social researcher should include updated income criteria, 2012. Indian J Community Med 2013;38:185-6.

21. DeVivo MJ. Causes and costs of spinal cord injury in the United States. Spinal Cord 1997;35:809-13.

22. Cifu DX, Huang ME, Kolakowsky-Hayner SA, Seel RT. Age, outcome, and rehabilitation costs after paraplegia caused by traumatic injury of the thoracic spinal cord, conus medullaris, and cauda equina. J Neurotrauma 1999;16:805-15.

23. Margolis JM, Juneau P, Sadosky A, Cappelleri JC, Bryce TN, Nieshoff EC. Health care resource utilization and medical costs of spinal cord injury with neuropathic pain in a commercially insured population in the United States. Arch Phys Med Rehabil 2014;95:2279-87.

24. Webster B, Giunti G, Young A, Pransky G, Nesathurai $S$. Work-related tetraplegia: cause of injury and annual medical costs. Spinal Cord 2004;42:240-7.

25. SCI facts and figures 2016. J Spinal Cord Med 2016;39:737-8. 